\title{
(2) Adaptive trial designs: what are OPEN ACCESS multiarm, multistage trials?
}

\author{
Gerard Cathal Millen (D) , ${ }^{1}$ Christina Yap (D) ${ }^{2,3}$
}

\begin{abstract}
${ }^{1}$ Paediatric Oncology, Birmingham Women's and Children's NHS Foundation Trust, Birmingham, UK

${ }^{2}$ Cancer Research UK Clinical Trials Unit, Institute of Cancer and Genomic Sciences, University of Birmingham College of Medical and Dental Sciences, Birmingham, UK ${ }^{3}$ Clinical Trials and Statistics Unit, Institute of Cancer Research, London, UK

\section{Correspondence to}

Dr Gerard Cathal Millen, Paediatric Oncology, Birmingham Women's and Children's NHS Foundation Trust, Birmingham, B4 6NH, UK; g.millen@nhs.net
\end{abstract}

Received 9 August 2019 Revised 11 October 2019 Accepted 18 October 2019

\section{Check for updates}

(C) Author(s) (or their employer(s)) 2020. Re-use permitted under CC BY. Published by BMJ.

To cite: Millen GC, Yap C. Arch Dis Child Educ Pract Ed Epub ahead of print: [please include Day Month Year]. doi:10.1136/ archdischild-2019-317826

\section{INTRODUCTION}

Clinical trials can be separated into phase I (dose finding and safety), phase II (activity or early efficacy), phase III (efficacy compared with current standard of care) and occasionally phase IV (postmarketing studies). A new compound would usually have to go through phase I-III studies sequentially with all of the financial and regulatory hurdles this poses. A recent study has estimated that only $13.8 \%$ of compounds tested will be successful in achieving a marketing license. ${ }^{1}$

Adaptive designs are an extensive class of flexible tools which use accumulated data in the trial to make preplanned adaptations to the trial's course. They can be used in all trial phases. The adaptations can include stopping an arm early for futility or safety, closing recruitment to an arm early if there is strong evidence of efficacy, changing of target sample size or allocation ratios. They are usually more efficient, informative and ethical than traditional fixed designs (where no interim adaptations are permitted). They can often offer savings in resources and even number of patients (figure 1$)^{2}$

A novel paradigm for conducting adaptive trials, which allows several treatments to be assessed concurrently with preplanned interim adaptations, is the so-called multiarm, multistage (MAMS) design. We will review the pros and cons of MAMS trials in this article.

\section{MULTIARM, MULTISTAGE TRIALS}

Due to high failure rates, substantial cost and time required, novel trial methodologies are required to streamline the pipeline of drugs from preclinical work to proven treatments. One such adaptive design is the MAMS trial. MAMS trials were first reported over 20 years ago as a way to accelerate the process of drug development. ${ }^{3}$
MAMS has been more commonly implemented in phase II/III settings, although it can be applied in any trial phase. Rather than a series of separate phase II/III studies, MAMS trials aim to answer multiple questions simultaneously under the same regulatory framework. Multiple different treatment options can be compared simultaneously, often against a control arm. ${ }^{4}$ These can either be different drugs/combinations of drugs or different doses of the same drug. Through the use of interim analyses with predetermined adaptation rules, different arms can be modified or even closed to further recruitment to focus the number of patients more on drugs which are showing good efficacy. ${ }^{5}$

One of the best-known examples of a MAMS trial is the STAMPEDE (Systemic Therapy in Advancing or Metastatic Prostate Cancer: Evaluation of Drug Efficacy) trial which looked at treatment of men with advanced or metastatic prostate cancer. The trial initially opened in 2005 with six arms (one standard of care arm and five experimental arms) with two planned interim analyses. Since opening, the initial five experimental arms have closed and new arms have subsequently been added. Clear evidence emerged that the addition of docetaxel to standard chemotherapy improved the survival of patients which resulted in the protocol being amended to allow the use of docetaxel as standard of care going forward from 2016 (figure 2).

Another example of a seamless phase II/ III MAMS trial is a paediatric rare cancer trial called rEECur (ISRCTN 36453794). This trial aims to identify the optimal treatment for relapsed/refractory Ewing's sarcoma, by comparing four commonly used chemotherapy regimens, with a drop-a-loser approach. ${ }^{6}$ There are two preplanned interim analyses, where the least promising arm (based on objective response rate) will be dropped after each 


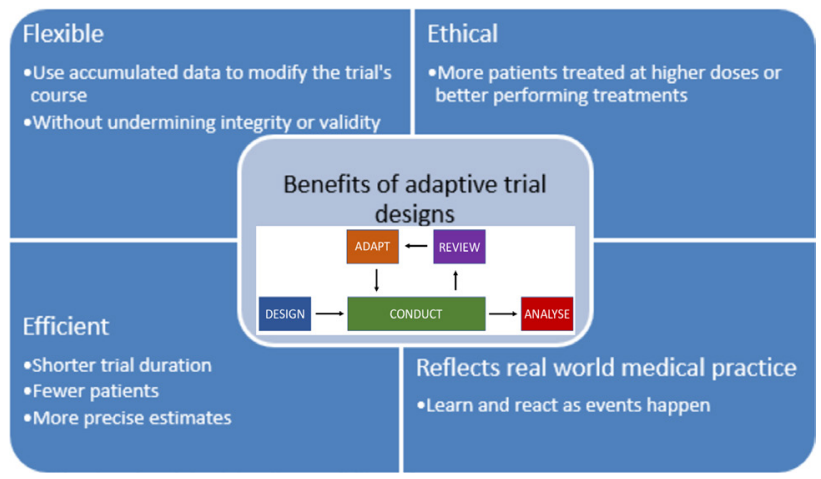

Figure 1 Benefits of adaptive trial designs. Adapted from: adaptive designs in clinical trials: why use them, and how to run and report them.

stage. In the final stage, the trial will proceed seamlessly to phase III comparing the two best remaining arms (based on progression-free survival).

MAMS trials have also been proposed as potentially attractive designs for studies in multidrug-resistant tuberculosis and $\mathrm{HIV}^{7}$

\section{BENEFITS AND RISKS OF MAMS TRIALS}

The biggest advantage of a MAMS trial is the ability to answer multiple research questions simultaneously under a single trial protocol and regulatory framework rather than answer them sequentially or via a series of separate trials as in the traditional paradigm. The latter will require a longer period of time, substantial higher costs as well as potentially larger number of patients.

Furthermore, using amendments to trial protocols, MAMS can easily lend itself to a platform design where new arms can be added while the study is ongoing. This is much more efficient than designing a new trial. ${ }^{8}$

MAMS trials can also be considered more ethical and beneficial for patients as they reduce the number of patients treated at ineffective doses or with ineffective or harmful drugs as well as maximising the numbers treated on more efficacious arms (figure 1).

There are some limitations of, or risks associated with MAMS trials. Due to the smaller number of patients at the interim analyses, there is a risk that potentially efficacious treatments may be rejected if they meet the predefined stopping rules. 5910

In addition, MAMS trials can be more resource intensive in the initial design phase and during trial conduct as there are multiple arms and multiple stages. The increased complexity may result in clinicians being less keen on using them due to lack of awareness or need for greater specialist input from statisticians. ${ }^{10}$

Although adaptive designs, such as MAMS, often provide notable efficiency benefits, there are situations where they may not be worthwhile. It is vital that careful consideration of key factors (including outcomes, recruitment, data quality and trial complexity) should be built into deciding and developing the most appropriate trial design to answer the trial's objectives. ${ }^{10}$

\section{CONCLUSIONS}

Historically, clinical trials have been designed using traditional phase I-III methodology with sequential or separate trials that can take many years and have a low overall success rate in delivering drugs to patients.

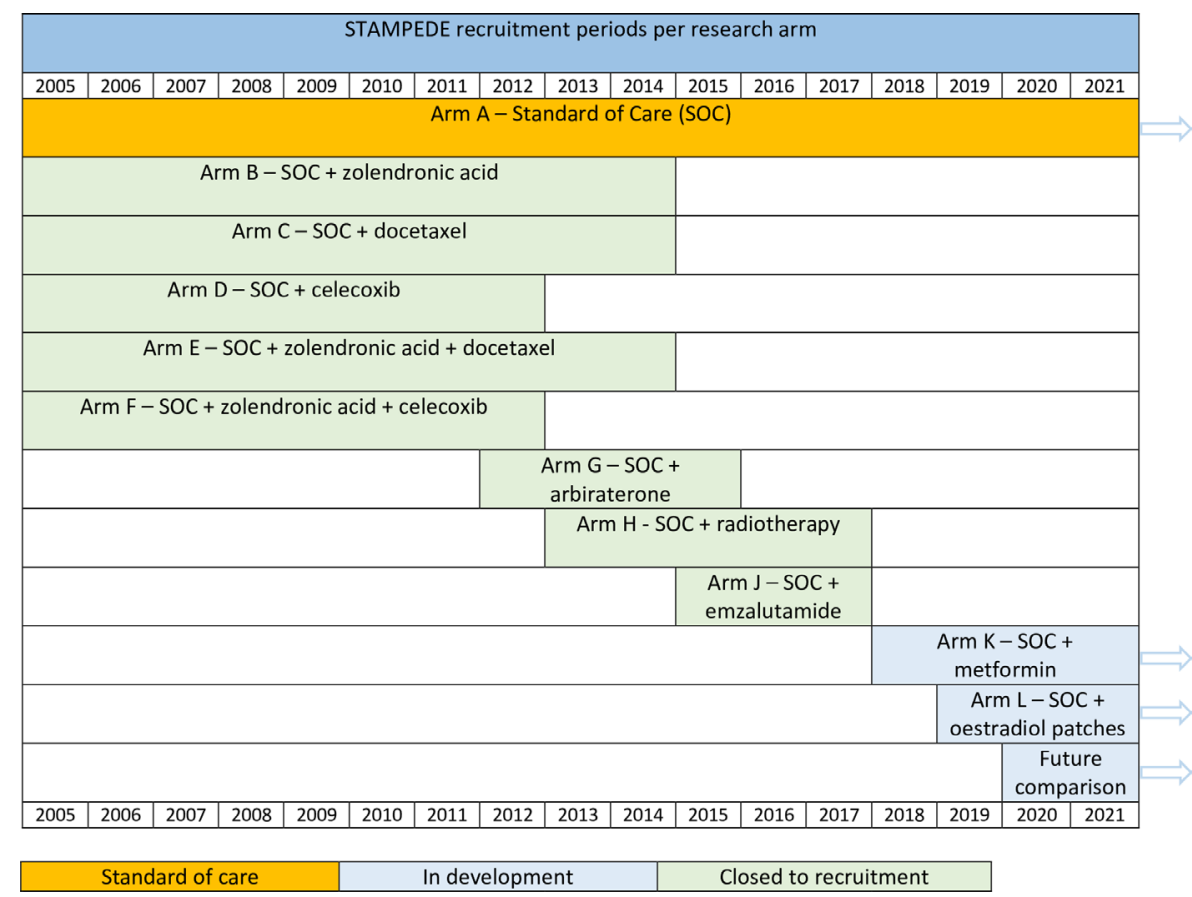

Figure 2 Schematic diagram of STAMPEDE trial. Adapted from: this is a platform alteration: a trial management perspective on the operational aspects of adaptive and platform and umbrella protocols. ${ }^{8}$ 
MAMS trials aim to overcome many of these difficulties by combining multiple experimental arms into the same study. Through the use of interim analyses, MAMS trials can be more efficient and ethical by removing non-performing arms earlier and channelling more patients into the most efficacious arms. Although not widely used in paediatrics yet, some trials are being designed and run in this fashion. It is likely to increase further in the future.

\section{Twitter Christina Yap @ChristinaBYap}

Contributors GCM wrote and revised the initial manuscript. CY wrote, reviewed and revised the manuscript prior to submission. Both authors approved the final manuscript.

Funding CY is funded by Cancer Research UK Grant No. C22436/A15958.

Competing interests None declared.

Patient consent for publication Not required.

Provenance and peer review Commissioned; externally peer reviewed.

Open access This is an open access article distributed in accordance with the Creative Commons Attribution 4.0 Unported (CC BY 4.0) license, which permits others to copy, redistribute, remix, transform and build upon this work for any purpose, provided the original work is properly cited, a link to the licence is given, and indication of whether changes were made. See: https:// creativecommons.org/licenses/by/4.0/.

\section{ORCID iDs}

Gerard Cathal Millen http://orcid.org/0000-0003-1446-3228

Christina Yap http://orcid.org/0000-0002-6715-2514

\section{REFERENCES}

1 Wong $\mathrm{CH}$, Siah KW, Lo AW. Estimation of clinical trial success rates and related parameters. Biostatistics 2019;20:273-86.

2 Pallmann P, Bedding AW, Choodari-Oskooei B, et al. Adaptive designs in clinical trials: why use them, and how to run and report them. BMC Med 2018;16.

3 Royston P, Parmar MKB, Qian W. Novel designs for multi-arm clinical trials with survival outcomes with an application in ovarian cancer. Stat Med 2003;22:2239-56.

4 Ghosh P, Liu L, Senchaudhuri P, et al. Design and monitoring of multi-arm multi-stage clinical trials. Biometrics 2017;73:1289-99.

5 Lin J, Bunn V. Comparison of multi-arm multi-stage design and adaptive randomization in platform clinical trials. Contemp Clin Trials 2017;54:48-59.

6 Moroz V, Wheatley K, McCabe M. Flexible trial design in a rare condition. Trials 2013;14.

7 Kim S, Seddon JA, Garcia-Prats AJ, et al. Statistical considerations for pediatric multidrug-resistant tuberculosis efficacy trials. Int J Tuberc Lung Dis 2018;22:34-9.

8 Schiavone F, Bathia R, Letchemanan $\mathrm{K}$, et al. This is a platform alteration: a trial management perspective on the operational aspects of adaptive and platform and umbrella protocols. Trials 2019;20:264.

9 Barthel FM-S, Parmar MKB, Royston P. How do multi-stage, multi-arm trials compare to the traditional two-arm parallel group design--a reanalysis of 4 trials. Trials 2009;10:21.

10 Wason JMS, Brocklehurst P, Yap C. When to keep it simple adaptive designs are not always useful. BMC Med 2019;17. 